Meta

Journal des traducteurs

Translators' Journal

\title{
Logique naturelle et interprétation sémantique
}

\section{Antonio A. M. Querido}

Volume 18, numéro 1-2, mars 1973

Actes du deuxième colloque international de linguistique et de traduction. Montréal, 4-7 octobre 1972

URI : https://id.erudit.org/iderudit/003884ar

DOI : https://doi.org/10.7202/003884ar

Aller au sommaire du numéro

Éditeur(s)

Les Presses de l'Université de Montréal

ISSN

0026-0452 (imprimé)

1492-1421 (numérique)

Découvrir la revue

Citer cet article

Querido, A. A. M. (1973). Logique naturelle et interprétation sémantique. Meta, 18(1-2), 35-46. https://doi.org/10.7202/003884ar d'utilisation que vous pouvez consulter en ligne.

https://apropos.erudit.org/fr/usagers/politique-dutilisation/ 


\section{Logique naturelle et interprétation sémantique *}

\section{POUR RENDRE COMPTE DE CERTAINES OPPOSITIONS SÉMANTIQUES IL FAUT INTRODUIRE DES PRÉSUPPOSITIONS LOGIQUES \\ DANS L'ANALYSE SYNTAXIQUE}

Considérons les triplets d'exemples suivants:

(1) a) Marie a choisi Paul.

b) Celui que Marie a choisi (c') est Paul.

c) C'est Paul que Marie a choisi.

(2) a) Marie a choisi Paul.

b) Celle qui a choisi Paul (c')est Marie.

c) C'est Marie qui a choisi Paul.

(3) a) Marie a rencontré Paul à Paris.

b) L'endroit où Marie a rencontré Paul (c')est Paris.

c) C'est à Paris que Marie a rencontré Paul.

Pour chaque triplet considérons les exemples a) et c). Devant ces couples d'exemples les linguistes ont adopté deux attitudes radicales : 1) la première que nous appellerons le «structuralisme asémantique » prétend qu'il n'y a pas de relation formelle entre a) et c) : il s'agirait de deux structures syntaxiques complètement indépendantes ; 2) la seconde attitude, le «transformationalisme asémantique », prétend que a) et c) sont reliés par des transformations et qu'ils

* Cette étude a été réalisée dans le cadre du projet de recherche a Linguistique et intelligence artificielle », subventionné par le Conseil des arts du Canada, Division des humanités et des sciences sociales, et par l'Université de Montréal. Le but de ce projet est de construire un programme capable d' "interpréter » des phrases du français dans un domaine restreint. Appelons ce programme interprète. Imaginons un microcosme contrôlé par un robot auquel on a associé un programme de démonstration de théorèmes, destiné à résoudre les problèmes qui se posent à lui dans son environnement. Pour assurer la communication du robot avec un interlocuteur humain il faut lui associer un interprète (dans le jargon de l'informatique un "compilateur») d'une langue naturelle. L'interprète doit contenir un ensemble de règles de syntaxe, de sémantique et de logique naturelle (une "grammaire générative »). Mais cela n'est pas suffisant. Comme Chomsky l'a montré, une grammaire générative n'est qu'un modèle de la compétence linguistique. Pour pouvoir "comprendre " des phrases du français il faut que l'interprète soit pourvu de stratégies d'analyse et qu'il puisse avoir recours, à tout instant du processus d'interprétation, à des connaissances extra-linguistiques. Qu'il s'agisse d'un ordre, d'une question ou d'une déclaration, il ne pourra les interpréter pleinement que par rapport aux objets, aux événements et aux changements du microcosme. Nous croyons que c'est là la meilleure façon de tester l'adéquation et la puissance d'une grammaire générative. Il va sans dire qu'une grammaire générative ne saurait décrire qu'un fragment du français et que sa puissance ne pourra être mesurée que par rapport aux hypothèses linguistiques posées au départ. 
sont par conséquent des paraphrases. Nous essayerons d'éviter ces attitudes extrêmes et concéderons au "transformationalisme asémantique » qu'il y a une relation formelle entre a) et c) mais que ce n'est pas une relation de paraphrase pure.

La meilleure façon de montrer la différence entre a) et c) c'est d'introduire la notion logique de "présupposition». Quand le locuteur affirme (1) c) il présuppose que Marie a choisi quelqu'un. Une situation appropriée pour l'usage d'une construction "c'est que » est l'annonce de l'élection d'un pape par le conclave. La fumée blanche a déjà introduit la présupposition « Le conclave a choisi $x »$. Le cardinal qui annonce le nom du nouveau pape peut donc dire «C'est $x_{1}$ que le conclave a choisi ». La structure profonde ou forme logique de (1) a) contient donc deux propositions ou si l'on préfère une présupposition et une proposition :

(4) (3x) Marie a choisi $x$. Ce $x$ est Paul.

Il est bien connu que l'on peut transformer une suite de deux phrases reliées par un adjectif démonstratif (anaphorique) en une phrase complexe dans laquelle la première phrase devient une proposition relative de la seconde :

(5) a) Marie a rencontré un garçon hier.

Ce garçon est très brillant.

b) Le garçon que Marie a rencontré hier est très brillant.

Cette transformation, l'enchassement de la relative, permet de dériver (6) de (4) :

(6) Ce $x$ que Marie a choisi est Paul.

Puisque le $x$ en l'occurrence se réfère à un humain on peut traduire en français (6) par (1) b). Pour démontrer que (1) b) est une structure intermédiaire dans la dérivation transformationnelle de (1) c) il suffit d'établir qu'il y a une règle en français qui relie systématiquement les constructions du type b) à celles du type c).

La transformation d'extraposition est assez fréquente en français et dans d'autres langues. Elle prend une proposition subordonnée qui se trouve au début ou au milieu d'une phrase et la transporte à la fin de cette phrase :

(7) a) Que Marie ne soit pas là est surprenant (pour Paul).

b) Il est surprenant (pour Paul) que Marie ne soit pas là.

(8) a) *J'ai annoncé que Marie n'était pas là à Paul.

b) Je l'ai annoncé à Paul.

c) J'ai annoncé cela à Paul.

d) J'ai annoncé à Paul que Marie n'était pas là.

(9) a) Un monsieur qui voulait te parler est venu.

b) Un monsieur est venu qui voulait te parler.

(10) a) Un monsieur qui veut te parler est là.

b) Un monsieur est là qui veut te parler. 
Remarquons que dans (7) la proposition extraposée, puisqu'elle occupait la position sujet, doit être remplacée par un sujet explétif. Tel n'est pas le cas dans les extrapositions de (8), (9) et (10). L'insertion du sujet explétif est une exigence des langues à sujet comme l'anglais et le français. Dans les langues comme l'espagnol et le portugais la proposition sujet est extraposée sans laisser de remplaçant.

(11) a) Que a Maria não esteja cá é surpreendente.

b) E surpreendente que a Maria não esteja cá.

Si la phrase de (8) a) n'est pas grammaticale il faut conclure que l'extraposition est obligatoire dans ce type de contexte. Remarquons que la proposition à extraposer est bien l'objet de la phrase $(8)$ a) comme le montrent les phrases $(8)$ b) et c) ou «le» et «cela » peuvent avoir comme antécédent «Marie n'est pas là ».

Les exemples (9) et (10) montrent qu'au moins dans certains contextes une proposition relative peut aussi être extraposée. Si l'on fait abstraction de l'insertion du sujet explétif et des restrictions contextuelles qui commandent l'obligatoriété ou l'optionalité de l'extraposition on peut la formuler de façon très générale comme suit :

$$
\begin{array}{ccc}
{ }_{P} X & P & \left.Y_{P}\right] \\
1 & 2 & 3 \\
1 & \theta & 3+2
\end{array} \rightarrow
$$

Dans cette formule $P$ représente une proposition enchassée dans une phrase supérieure; $X$ et $Y$ sont des variables qui représentent des suites de nœuds syntagmatiques de longueur quelconque (y inclus la suite vide). La transformation a comme résultat de détacher de l'arbre l'élément qui a l'indice 2 et de l'attacher à droite de l'élément qui a l'indice 3.

La transformation (12), nécessaire par ailleurs pour décrire d'autres structures de surface du français, est le lien recherché pour relier les exemples b) et c) des triplets (1), (2) et (3).

Il reste évidemment quelques petits problèmes à résoudre comme le double démonstratif de (1) b) et le remplacement de «celui» par «ce» dans (1) c). Mais en gros si on applique la règle d'extraposition à (6) on dérive :

$$
\text { Ce }(x) \text { est Paul que Marie a choisi. }
$$

En résumé, nous avons essayé de démontrer que la différence entre (1) b) et c) d'une part et (1) a) d'autre part était que la structure profonde de (1) b) et c) contenait une présupposition telle que représentée dans (4).

Remarquons que de (1) a) on peut également déduire la première proposition de (4). Si vous affirmez que Marie a choisi Paul, alors, vous répondrez vraisemblablement par «oui » à la question «Est-ce que Marie a choisi quelqu'un? ». 
Malgré cela la première proposition de (4) n'est pas une présupposition, au sens logique, de (1) a). La présupposition se maintient en effet sous la négation et sous l'interrogation. Or, voyons ce qui se passe si l'on nie ou interroge (1) a) et (1) c):

(14) a) Marie n'a pas choisi Paul.

b) Est-ce que Marie a choisi Paul ?

(15) a) Ce n'est pas Paul que Marie a choisi.

b) Est-ce Paul que Marie a choisi?

De (15) a) et b) on peut encore conclure que le locuteur croit (présuppose) que Marie a choisi quelqu'un mais pas de (14) a) et b).

\section{POUR UNE INTERPRÉTATION SÉMANTIQUE ADÉQUATE ON A BESOIN D'INTRODUIRE DANS L'ANALYSE DES VARIABLES ET DES QUANTIFICATEURS}

Examinons les exemples suivants :

(16) a) Jean croit avoir raison.

b) Jean $x_{x_{1}}$ croit qu'il $x_{1}$ a raison.

c) *Jean croit que Jean ${ }_{x_{1}}$ a raison.

Nous avons attaché à quelques couples de termes une variable indexée pour indiquer qu'ils ont le même référent. Cela veut dire qu'à propos de la forme «Jean croit qu'il a raison» nous voulons parler seulement de l'interprétation sémantique dans laquelle «Jean » est l'antécédent de «il ». Dans ce cas (16) c) n'est pas grammaticale.

La règle à dégager serait donc que si la proposition-complément de «croire » et la proposition principale ont des sujets co-référents alors le sujet de la proposition-complément doit être élidé [cf. (16) a)] ou pronominalisé [cf. (16) b)]. Il ne suffit pas que les sujets aient la même forme comme le prouve l'exemple suivant :

$$
\text { Ce garçon }{ }_{x_{1}} \text { croit que ce garçon }{ }_{x_{1}} \text { est avant lui }{ }_{x_{1}}
$$

Nous supposons que «ce» est un déictique et que le locuteur peut utiliser des gestes pour indiquer le référent de chaque occurrence de «ce garçon». Le rôle des gestes déictiques peut être considéré comme équivalent des variables indexées que nous introduisons artificiellement pour noter les diverses interprétations sémantiques.

Examinons maintenant à la lumière de cette règle ce qui se passe dans les exemples suivants :

(18) a) Tous les linguistes croient avoir raison.

b) Tous les linguistes croient qu'ils ont raison.

c) Tous les linguistes croient que tous les linguistes ont raison.

Il est évident que (18) a) et b) ne s'interprètent pas de la même façon que (18) c). Par ailleurs, la discussion antérieure en termes de coréférence de syntagmes nominaux ne semble pas pertinente. 
La seule façon de représenter adéquatement cette opposition sémantique semble être une notation logique qui possède, en plus des variables, des quantificateurs.

Introduire des quantificateurs dans l'analyse grammaticale d'une langue naturelle revient à postuler qu'à un certain niveau de profondeur «tous » ne fait pas partie du syntagme nominal mais modifie toute la phrase. C'est-à-dire, qu'avant de devenir un adjectif déterminatif en syntaxe de surface «tous» est un opérateur de la phrase en syntaxe de profondeur :

(19) a) $x \in[x:$ linguiste $(x)]$

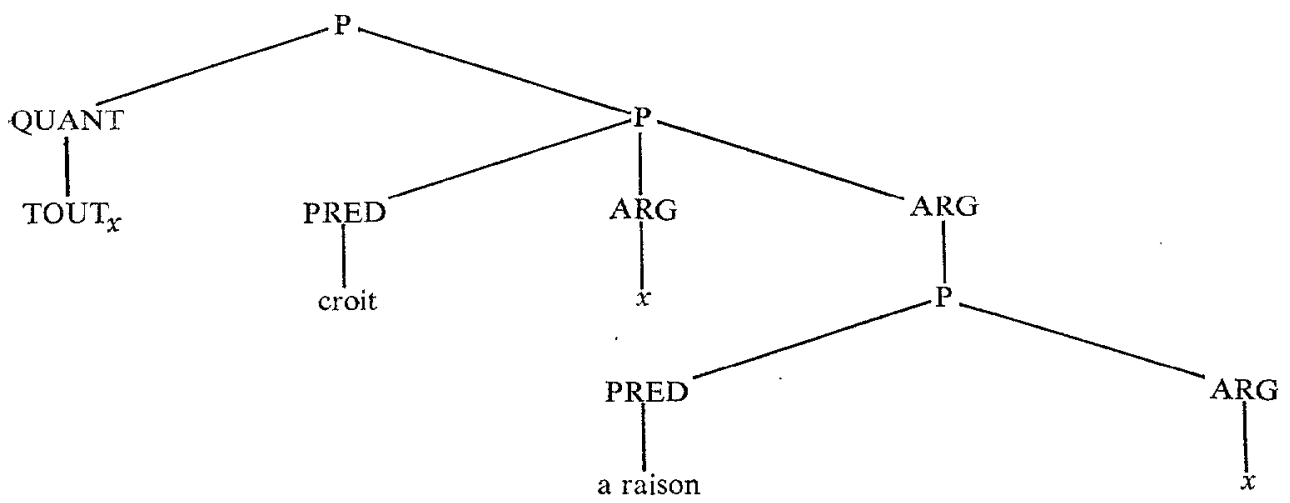

b) $x \in[x$ : linguiste $(x)]$

$y \in[y$ : linguiste $(y)]$

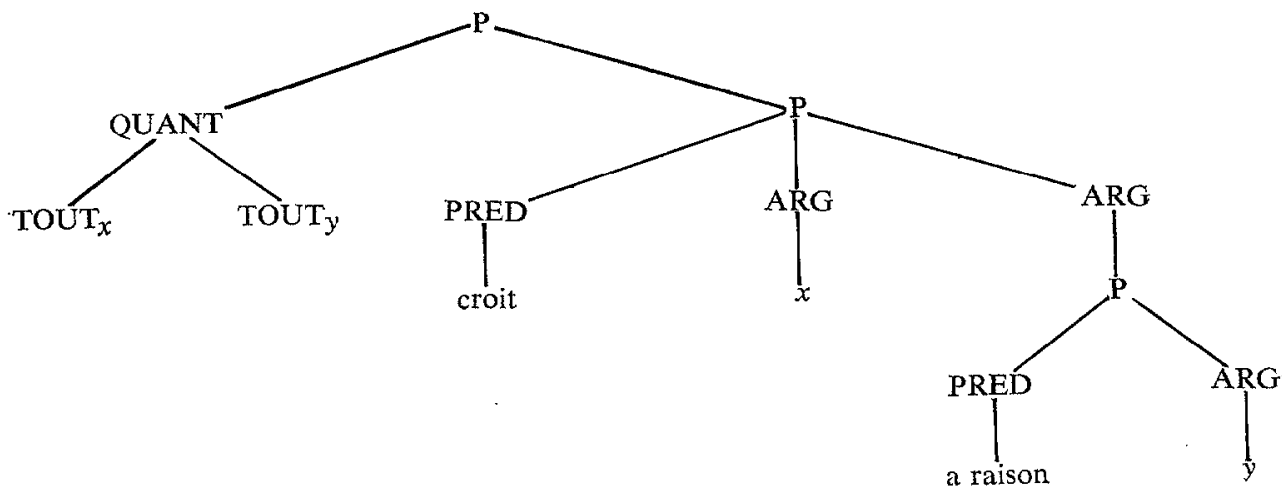

La structure (19) a) peut se réaliser en surface sous la forme (18) a) ou (18) b), tandis que de (19) b) on peut dériver (18) c).

Cette analyse explique pourquoi le sujet de «a raison» peut être élidé ou pronominalisé selon la règle évoquée auparavant dans (19) a) mais pas dans (19) b). Elle explique aussi que en paraphrasant (18) c) on introduit normalement deux termes (d'ici le besoin de deux variables dans la structure profonde) :

(20) a) Tout linguiste croit que tout autre linguiste et lui-même ont raison.

b) Quels que soient $x$ et $y$, si $x$ et $y$ sont des linguistes, alors $x$ croit que $y$ a raison (même dans le cas ou $x=y$ ). 
Le changement de rôle syntaxique de \& tous » (de quantificateur en syntaxe logique il devient adjectif déterminatif en syntaxe de surface) exige que la grammaire contienne une règle d'abaissement $d u$ quantificateur qui le détache de sa position supérieure et l'attache à l'argument (syntagme nominal) qui domine la variable liée. Il est évident que l'élision de $\mathrm{SN}$ identique ou la pronominalisation doivent s'appliquer auparavant pour détruire le second $x$. Cela expliquera pourquoi dans (18) a) et b) il y a un seul «tous » tandis que dans (18) c) il y en a deux.

L'introduction des quantificateurs en syntaxe de profondeur permet également d'expliquer nombre de déductions en langue naturelle. Ainsi examinons (21) et (22) :

(21) a) Toutes les théories sont indéfendables.

b) Il n'y a pas de théorie défendable.

(22) a) Toutes les théories ne sont pas défendables.

b) Il y a des théories indéfendables.

La syntaxe de surface de ces deux couples ne permet pas d'expliquer comment a) et b) sont équivalents sémantiquement (paraphrases) dans (21) et dans (22) tandis que (21) a) et (22) a) ne le sont pas. Pourtant (21) a) et (22) a) sont tous les deux négatifs, ont tous les deux le même sujet (toutes les théories) et le même prédicat (sont défendables). La négation est dans un cas attachée au prédicat sous la forme d'un préfixe (in-) et dans l'autre sous la forme d'un adverbe de négation (ne pas).

Ce que la syntaxe de surface ne révèle pas c'est que dans (21) a) la négation modifie directement la proposition gouvernée par «sont défendables », tandis que dans (22) a) elle modifie directement la phrase gouvernée par \& toutes ». Les relations sémantiques ou logiques sous-jacentes à (22) a) seraient mieux révélées par (23) si c'était une phrase grammaticale :

(23) ?Pas toutes les théories sont défendables.

Si en plus des quantificateurs tout et $y$ a nous introduisons l'unité sémantique non comme opérateur de propositions ou prédicat supérieur nous serons en mesure d'expliquer les règles de déduction qui permettent de relier sémantiquement (21) a) à (21) b) et (22) a) à (22) b).

Analysons donc ces trois opérateurs comme des prédicats (PRED) qui prennent des propositions ( $\mathrm{P}$ ) comme arguments. Le schéma d'une proposition négative sera donc (24) et celui d'une proposition quantifiée (25) :

(24)

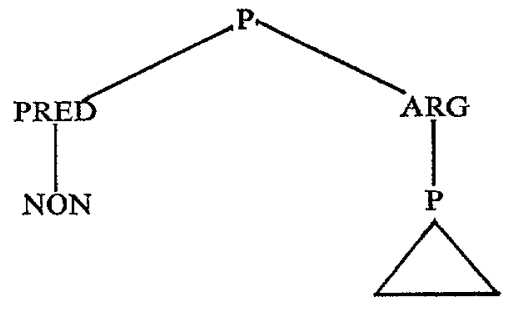

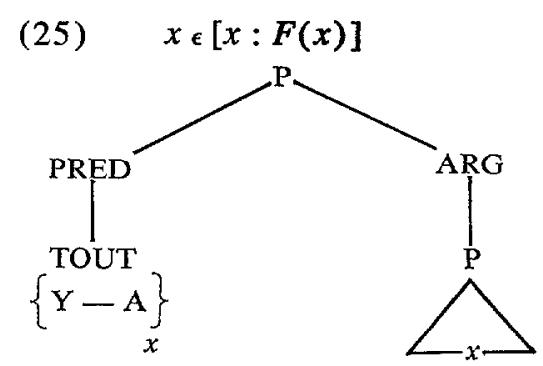


Remarquons d'abord l'équivalence logique de la double négation qui permet de généraliser le cas du couple de phrases suivantes :

(26) a) Il y a des théories qui ne sont pas indéfendables.

b) Il y a des théories (qui sont) défendables.

(27) Élision de la double négation :

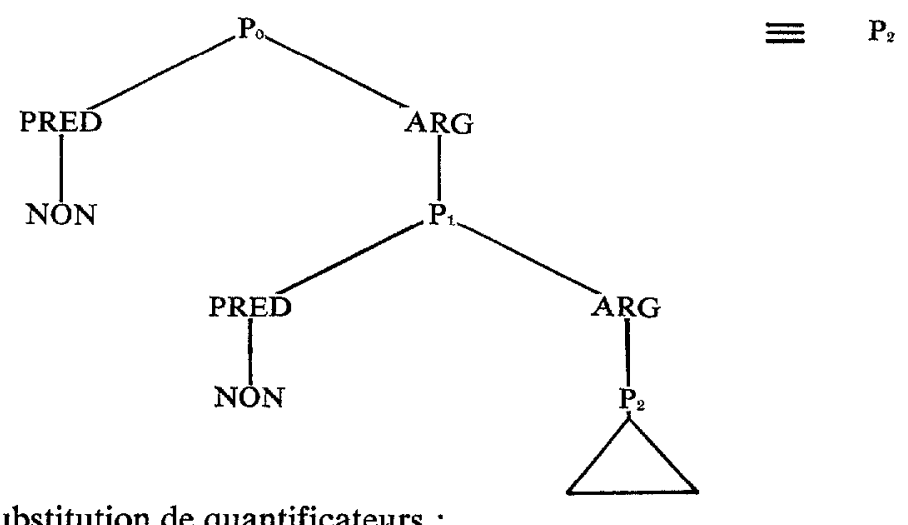

(28) Substitution de quantificateurs :

a)
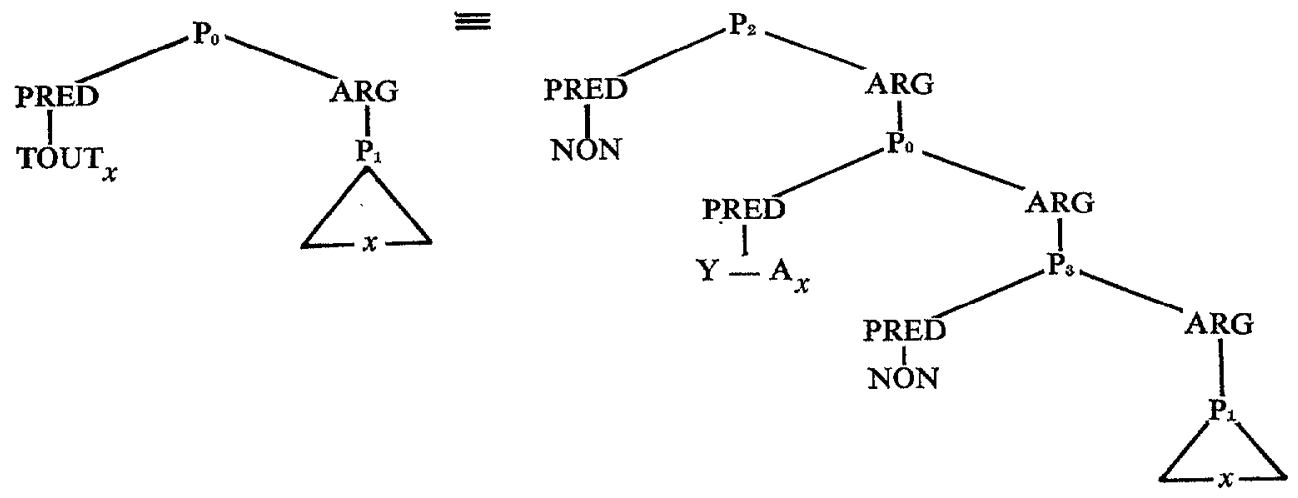

b)
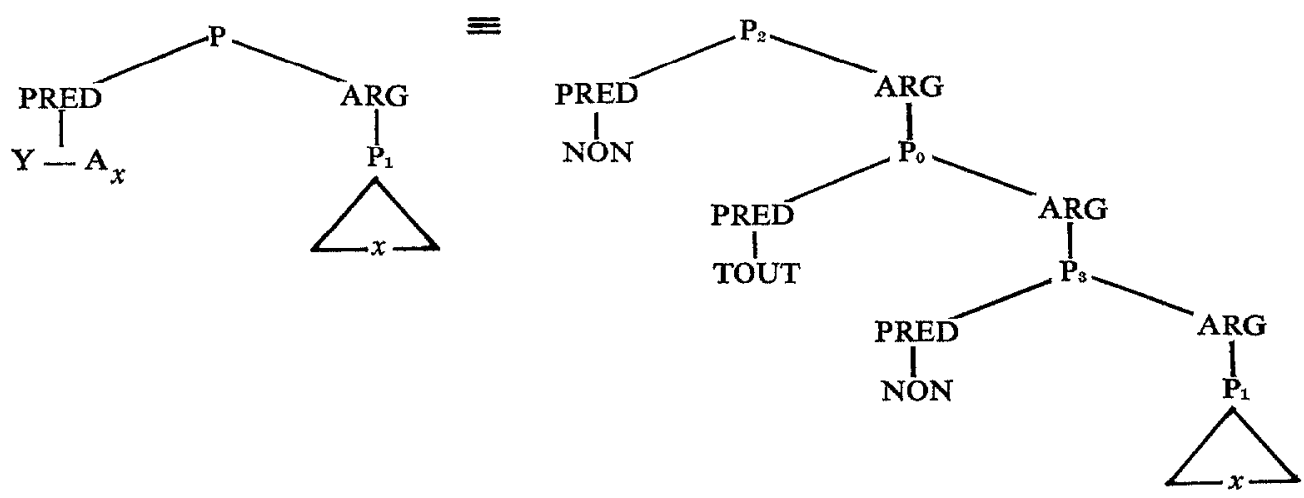
Les règles (27) et (28) permettent d'expliquer toutes les équivalences sémantiques du type de (21) a) et b) et (22) a) et b).

Une telle explication serait impossible ou du moins extrêmement complexe si on analysait «tout » comme adjectif déterminatif ou pré-article et «non », «ne... pas » ou « in-» comme adverbes de négation ou pré-phrases (c'est le cas des structures profondes proposées dans le cadre du modèle standard de grammaire transformationnelle).

De telles structures ne sont pas assez profondes parce qu'elles ne révèlent pas toutes les relations syntaxiques pertinentes pour l'interprétation sémantique de façon uniforme et non ambiguë.

Remarquons que le schéma de la règle (28) s'applique aussi à d'autres couples de prédicats. Ainsi les prédicats nécessaire et possible (dans l'interprétation dite de nécessité et de possibilité logiques) se comportent de façon identique sous la négation :

(29) a) L'ami de ton ami n'est pas nécessairement ton ami.

b) Il est possible que l'ami de ton ami ne soit pas ton ami.

La règle (28) devra donc être généralisée à tous les couples de prédicats qui jouissent de cette dualité sous la négation. Ainsi pour tout couple de prédicats $(\tau, \psi)$ qui appartient à cette classe nous aurions la règle $(15)$ :

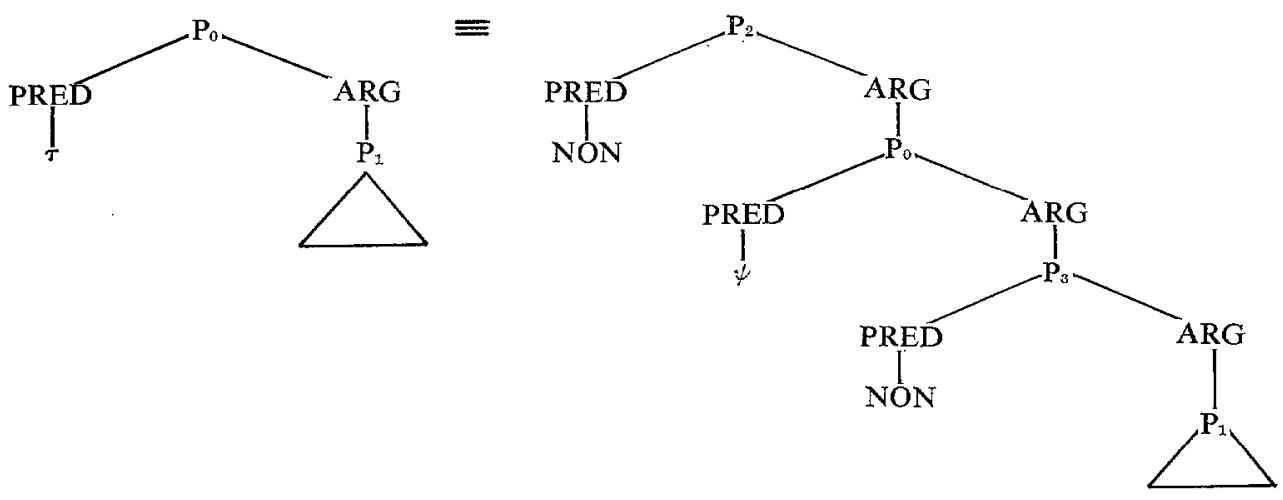

La restriction additionnelle selon laquelle, dans le cas des quantificateurs universel et existentiel, $P_{1}$ doit contenir la variable liée est indépendante de cette règle.

III. LES CATÉGORIES ET LES RELATIONS SYNTAXIQUES PERTINENTES POUR L'INTERPRÉTATION SÉMANTIQUE NE SONT PAS LES CATÉGORIES ET RELATIONS DE LA SYNTAXE DE SURFACE

Que la substitution $\tau-\psi$ fasse partie des règles de transformation ou des axiomes de déduction logique, une chose est certaine : sa formulation n'est possible que si le prétendu adverbe de négation, l'adjectif déterminatif «tout», la 
locution verbale «y $\mathrm{a} »$, l'adverbe «nécessairement» et l'adjectif «possible » sont «traduits» en syntaxe logique. Les logiciens appellent ces éléments des «opérateurs ». Mais, les opérateurs se comportent comme les «prédicats ». Il est certain en tout cas que les catégories syntaxiques de surface ne sont pas adéquates pour l'interprétation sémantique. Un prédicat logique, nous l'avons vu dans la discussion de plusieurs exemples, peut se réaliser en surface sous une forme verbale, adjectivale, adverbiale et même nominale.

La traduction d'une phrase en structure logique suppose donc une réduction des catégories syntaxiques de surface.

Les relations ou fonctions syntaxiques de surface ne sont pas non plus adéquates pour l'interprétation sémantique. Chomsky lui-même avait déjà remarqué que la relation sujet-prédicat de surface ne correspondait pas toujours à la relation sujet-prédicat de profondeur et que c'était la dernière qui était pertinente pour l'interprétation sémantique.

(31) a) Convaincre un linguiste c'est difficile.

b) Il est difficile de convaincre un linguiste.

c) Un linguiste est difficile à convaincre.

Ainsi des exemples (31) a) à c) c'est (31) a) qui se rapproche le plus de la structure profonde. Après Chomsky des théoriciens de la grammaire comme Fillmore, Lakoff et McCawley sont allés plus loin en rejetant la pertinence de la relation sujet-prédicat dans la représentation sémantique des phrases.

La linguistique a suivi en quelque sorte l'évolution de la logique en remplaçant l'analyse aristotélicienne par une analyse en termes de prédicats et d'arguments.

La fonction «sujet de la phrase», toujours pertinente en syntaxe de surface pour les langues qui connaissent la règle de formation du sujet, n'a pas d'équivalent en syntaxe logique. Ainsi un prédicat symétrique comme "voisin de n'a pas de préférence pour un de ses arguments :

(32) a) Jean est le voisin de Paul.

b) Paul est le voisin de Jean.

La représentation sémantique de ces deux exemples, c'est-à-dire leur traduction en syntaxe logique, devra neutraliser l'opposition résultant du choix d'un sujet en syntaxe de surface. En syntaxe logique le prédicat «voisin de» prend un ensemble d'arguments non ordonnés ou, si l'on préfère, un argument qui dénote un ensemble d'objets :

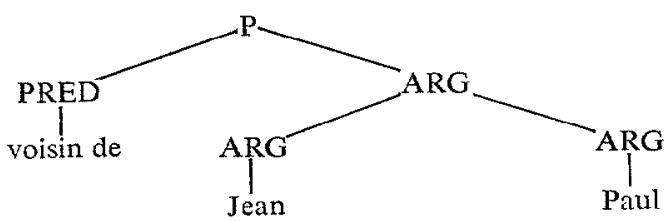

La structure de surface qui se rapproche le plus de cette structure logique est celle où il n'y a pas choix d'un « sujet de » la phrase : 
(34) Jean et Paul sont (des) voisins.

Même réflexion dans le cas des couples de prédicats converses :

(35) a) La maison de Jean est avant celle de Paul.

b) La maison de Paul est après celle de Jean.

Pour formaliser l'équivalence sémantique de (35) a) et b) il faut introduire une règle de substitution des prédicats converses. Comme la règle de substitution $\tau-\psi$ elle peut être considérée comme une transformation ou comme un axiome de déduction. Dans l'état actuel des sciences du langage nous ne disposons pas d'arguments pour trancher cette question.

La substitution des prédicats converses peut être formulée comme suit :

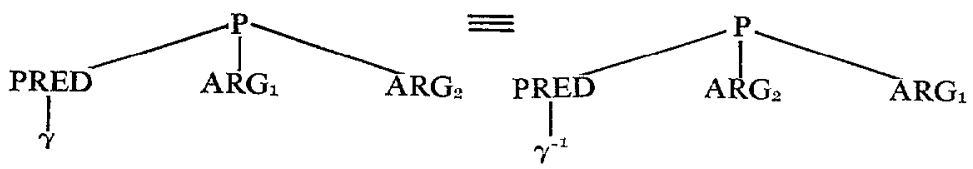

L'application de cette règle suppose la création d'un lexique des couples de prédicats converses. Chaque prédicat $\gamma$ peut être remplacé par converse $\gamma^{-1}$ si l'on intervertit ses arguments ${ }^{1}$.

La règle de formation de sujet que nous avons déjà mentionnée va choisir comme sujet l'argument qui est resté en première position après le choix de $\gamma$ ou $\gamma^{-1}$.

\section{LOGIQUE NATURELLE ET GRAMMAIRE}

L'interprétation sémantique des constructions que nous avons discutées, pour être adéquate, c'est-à-dire pour expliquer certaines équivalences et certaines oppositions sémantiques, exige que l'on analyse ces constructions en termes d'une syntaxe logique. Nous avons vu qu'il faut introduire dans cette syntaxe des éléments abstraits comme les présuppositions, les quantificateurs, les variables et l'opérateur de négation. Les catégories de la syntaxe de surface comme syntagme nominal (SN), verbe (V), adjectif (ADJ), déterminatif (DET), article (ART), pré-article (PRÉ-ART) et adverbe (ADV) doivent être réduites à des catégories plus abstraites comme prédicat (PRED) et argument (ARG). Les relations de la syntaxe de surface doivent être remplacées par des relations plus abstraites.

Certains éléments de la syntaxe de surface, comme l'auxiliaire copulatif « être », n'ont pas de représentant en syntaxe logique, n'étant pas nécessaires pour exprimer les relations sémantiques pertinentes. Ces éléments ne sont pas choisis en fonction de l'information sémantique que l'on veut coder dans une langue naturelle. En fait ils ne sont pas «choisis» mais imposés par la grammaire de cette langue.

1. Il ne s'agit pas exactement d'une interversion mais plutôt d'un transfert du premier argument en fin de phrase. C'est le mouvement que l'on observe aussi dans les transformations de nominalisation et de dérivation de la forme passive. 
De la même façon certains éléments de la syntaxe logique n'auront pas de représentant en syntaxe de surface. C'est évidemment le cas des variables référentielles qui sont remplacées systématiquement par un nom (en fait un prédicat qui peut prendre une forme nominale, par exemple «linguiste ») ou par un pronom. Ainsi dans la structure (19) a) le premier $x$ sera remplacé par «linguiste » et le second par un pronom anaphorique, tandis que dans (19) b) «linguiste $»$ remplacera $x$ et $y$.

La «traduction»d'une phrase en termes de structure logique est done gouvernée par des règles de transformation précises et systématiques.

Nous avons énuméré plusieurs règles et nous venons juste d'en mentionner deux autres qui font partie de la grammaire du français : l'insertion de l'auxiliaire copulatif ( être ») devant tous les prédicats qui ont une réalisation non verbale en structure de surface et le remplacement des variables référentielles.

Le rôle des transformations dans une grammaire est donc non seulement d' «engendrer» les phrases - c'est-à-dire d'introduire un critère formel de grammaticalité dans un système linguistique donné - mais aussi de systématiser la «traduction » de la forme de surface en une forme logique sous-jacente.

La forme de surface doit contenir toute l'information pertinente pour dériver un schéma phonétique et prosodique. La forme logique à son tour doit contenir toute l'information pertinente pour l'interprétation sémantique.

Il est évident que dans l'état actuel de la science nous ne connaissons pas tous les types d'information nécessaires pour une interprétation sémantique adéquate. Tout ce que nous avons essayé de faire c'est de soulever quelques oppositions et quelques équivalences sémantiques dont il faudra tenir compte dans l'analyse.

Pour systématiser le traitement de ces oppositions et de ces équivalences nous avons cru bon d'introduire dans l'analyse syntaxique des éléments, des catégories et des relations empruntées à la logique symbolique.

Cela n'implique pas que la logique des prédicats doive être intégrée telle quelle à la grammaire. Cela implique qu'une certaine logique naturelle doit être développée en fonction de l'interprétation sémantique des langues naturelles.

Tout le monde admettra que dans l'usage des mots «voisin», « avant» et « après » tout sujet parlant français sait tirer parti du caractère symétrique du premier et de la relation de prédicats converses des deux derniers. Les propriétés logiques des relations dénotées par les mots font donc partie de la compétence linguistique.

Jusqu'à présent la logique symbolique et la linguistique se sont développées de façon plus ou moins autonome. Pour créer un modèle adéquat d'interprétation sémantique il faut raccorder ces deux sciences du langage.

Le rôle spécifique de la linguistique sera de traduire des phrases en termes d'une structure profonde au moyen d'un système formel de transformations. Le rôle de la logique sera de construire à partir de cette structure profonde un système formel composé d'axiomes et de règles de déduction.

Le point de raccordement sara donc la structure profonde que toutes les deux doivent travailler à définir. 
En se pliant aux exigences de l'analyse des langues naturelles la logique symbolique deviendra véritablement logique naturelle. D'autre part l'analyse syntaxique doit se plier aussi aux exigences de la déduction logique. On aura ainsi à la place d'une syntaxe autonome à la Chomsky une syntaxe naturelle.

Antonio A.M. Querido

\section{BIBLIOGRAPHIE}

FILlmore, C. J., "Verbs of Judging : An Exercise in Semantic Description", dans Studies in Linguistic Semantics, C. J. Fillmore et D. T. Langendoen, édit., New York, Holt, Rinehart and Winston, 1971.

Harman, G., *Deep Structure as Logical Form », dans Semantics of Natural Language, D. Davidson et G. Harman, édit., Dordrecht, Reidel, 1972.

KartTunen, L., « Discourse Referents », 1971 (polycopié).

LAKofF, G., "The Role of Deduction in Grammar », dans Studies in Linguistic Semantics, C. J. Fillmore et D. T. Langendoen, édit., New York, Holt, Rinehart and Winston, 1971.

-, "Linguistics and Natural Logic », dans Semantics of Natural Language, D. Davidson et G. Harman, édit., Dordrecht, Reidel, 1972.

-, "On Generative Semantics ", dans Semantics : An Interdisciplinary Reader in Philosophy, Linguistics, Anthropology and Psychology, L. Jakobovits et D. Steinberg, édit., Cambridge, Cambridge University Press (sous presse).

McCawley, J., "A Program for Logic ", dans Semantics of Natural Language, D. Davidson et G. Harman, édit., Dordrecht, Reidel, 1972.

Millo, J., « Notes sur les réalisations de surface des quantificateurs et leur analyse », Groupe de recherche en linguistique formelle, Université de Montréal, 1972 (publication interne).

Querido, A. A. M., "Structures spatio-temporelles du français », Groupe de recherche en linguistique formelle, Université de Montréal, 1972 (publication interne).

-, "L'ordre polonais et les structures de base du français", dans Cahier de linguistique n० 2, Montréal, Les Presses de l'Université du Québec, 1972. 